\section{ORIGINAL RESEARCH}

D.F. Kallmes

B.A. Comstock

L.A. Gray

P.J. Heagerty

W. Hollingworth

J.A. Turner

L. Stout

J.G. Jarvik

\title{
Baseline Pain and Disability in the Investigational Vertebroplasty Efficacy and Safety Trial
}

BACKGROUND AND PURPOSE: Multiple case series of vertebroplasty outcomes have been published, though no large, placebo controlled trial has yet been performed. Our aim was to report baseline characteristics for the Investigational Vertebroplasty Efficacy and Safety Trial (INVEST), a randomized blinded controlled study of vertebroplasty.

MATERIALS AND METHODS: We compared baseline demographics, pain scores, and scores on the modified Roland-Morris Disability Scale (RMDS), a back pain-specific metric, between 2 groups. One group included subjects enrolled at the lead INVEST site ( $n=27$ to date). The second group consisted of eligible patients seen concurrently at the lead INVEST site, who declined enrollment $(n=70)$. Comparisons were made by using 2-sample $t$ tests.

RESULTS: Mean ages were similar between groups, averaging approximately 74 years among study participants and 77 years among nonenrolled eligible patients $(P=.17)$. Approximately $75 \%$ of subjects were female in both groups. RMDS scores of enrolled patients at the lead site (18.0 \pm 4.2$)$ were not statistically different from those of eligible nonenrolled patients at the lead site (18.6 \pm 3.6 , $P=.49$ ). Pain scores in the enrolled subjects were measured as "average intensity over the prior 24 hours" with mean scores of $7.6 \pm 2.1$ among enrolled patients at the lead site. Pain scores in eligible nonenrolled patients were measured as "pain at rest," with mean score of $3.4 \pm 3.3$, and "pain with activity," with mean score of $8.5 \pm 2.0$.

CONCLUSIONS: Patient demographics among subjects enrolled in the INVEST are similar to those in a cohort of eligible nonenrolled patients. Back pain-specific disability was similar between subjects enrolled in the INVEST study and eligible nonenrolled patients at the lead site.

$\mathbf{V}$ ertebroplasty is a widely applied procedure for palliation of pain from osteoporotic and neoplastic vertebral compression fractures. The procedure has been evaluated in numerous studies, including a large number of retrospective case series $^{1-8}$ and a relatively small number of nonrandomized controlled studies. ${ }^{9,10}$ These controlled studies typically focused on patients who underwent vertebroplasty or conservative management, usually with the potential for crossover in the short term. Only 1 randomized trial comparing vertebroplasty with medical therapy has been published. ${ }^{11}$ Almost without exception, these studies found large treatment effects from the procedure, though the treatment effect in the randomized trial was more modest than that in other reports. ${ }^{11}$

There is, to date, no blinded trial comparing vertebroplasty with a sham or placebo intervention. Such a study is needed to understand better the extent to which pain relief following vertebroplasty is due to a true treatment effect of the cement compared with other factors such as regression toward the mean, effects of local anesthesia, or nonspecific treatment effects, including patient and clinician expectations of pain relief.

Received November 18, 2008; accepted after revision December 22.

From the Department of Radiology (D.F.K., L.A.G.), Mayo Clinic College of Medicine, Rochester, Minn; Data Coordinating Center Comparative Effectiveness, Cost and Outcomes Research (B.A.C., P.J.H., J.A.T., L.S., J.G.J.), Center for Biomedical Statistics (B.A.C., P.J.H.), Departments of Psychiatry and Behavioral Sciences and Rehabilitation Medicine (J.A.T.), Departments of Radiology, Neurosurgery, and Health Services (J.G.J.), University of Washington, Seattle, Wash; and Department of Social Medicine (W.H.), University of Bristol, Bristol, UK.

This study was funded by National Institutes of Health (NIH) grant AR49373.

Please address correspondence to David F. Kallmes, MD, Mayo Clinic, 200 First St SW, Rochester, MN 55905; e-mail: Kallmes.david@mayo.edu

Indicates open access to non-subscribers at www.ajnr.org

DOI 10.3174/ajnr.A1519
The Investigational Vertebroplasty Efficacy and Safety Trial (INVEST) is a National Institutes of Health - sponsored international multicenter prospective randomized blinded trial comparing vertebroplasty with a "control intervention." 12 The control intervention consisted of a simulated vertebroplasty comprising all aspects of the typical vertebroplasty procedure except placement of the biopsy needle and cement. The study was designed to assess the true treatment effect of the cement in the vertebroplasty procedure compared with the other factors listed above.

The results of any clinical trial need to be interpreted in light of the study sample. Patient characteristics may influence response to any treatment and findings from a single clinical trial may or may not generalize to populations of patients with different demographic or clinical characteristics. Furthermore, patients who enroll in a randomized trial of a treatment may differ in important ways from patients who receive the treatment outside a research study. Therefore, the purpose of the present report was to describe the baseline demographic and clinical characteristics of patients who enrolled in the INVEST study and to compare them with those of patients who were eligible for the study but declined to enroll. This information will assist practitioners, researchers, and payers in interpreting vertebroplasty efficacy and safety results in the INVEST study when they are available.

\section{Materials and Methods}

Study Inclusion and Exclusion. Protocol details for INVEST are published in detail elsewhere. ${ }^{12}$ Institutional review board approval was obtained for this study, and all participants provided informed consent. Eligible patients are those with $\leq 3$ osteoporotic compression fractures of $<1$-year duration. MR imaging or bone scanning was used to characterize fractures in cases without serial plain radiographs 


\begin{tabular}{|c|c|c|c|}
\hline \multicolumn{4}{|c|}{$\begin{array}{l}\text { Patient demographics and baseline measures of patients enrolled in the INVEST study compared with those who were eligible for the study } \\
\text { but refused }\end{array}$} \\
\hline & $\begin{array}{l}\text { Lead Site, } \\
\text { Enrolled }(n=27)\end{array}$ & $\begin{array}{l}\text { Lead Site, } \\
\text { Eligible, Refused }(n=70)\end{array}$ & $P$ Value $^{*}$ \\
\hline \multicolumn{4}{|l|}{ Demographics } \\
\hline Age, mean (SD) (years) & $73.7(9.2)$ & $76.6(9.2)$ & 0.17 \\
\hline Women, №. $(\%)$ & $20(74)$ & $55(79)$ & 0.64 \\
\hline \multicolumn{4}{|l|}{ Primary measures, mean (SD) } \\
\hline RMDS score (0-23) & $18.0(4.2)$ & $18.6(3.6)$ & 0.51 \\
\hline \multicolumn{4}{|l|}{ Pain numeric rating scale $(0-10)$} \\
\hline At rest & & $3.4(3.3)$ & - \\
\hline With activity & & $8.5(2.0)$ & - \\
\hline Pain intensity (average in last 24 hours) $\dagger$ & $7.6(2.1)$ & & - \\
\hline
\end{tabular}

Note:--indicates not compared; RMDS, Roland-Morris Disability Scale.

${ }^{*}$ Age, proportion of women, and RMDS scores between INVEST subjects at the lead site and eligible/refused patients compared using 2-sample $t$ tests and $\chi^{2}$ tests (where appropriate). † INVEST study measure of pain.

to document fracture chronicity. Patients with fractures from neoplasms, including multiple myeloma and metastases, were excluded as were patients maintained on anticoagulation.

Procedural Details. Subjects were randomized 1:1 to either a regular vertebroplasty or a "control intervention." Vertebroplasty was performed in standard fashion, typically with unipediculate needle placement and deposition of barium-opacified polymethylmethacrylate, following skin and subcutaneous tissue infiltration with $1 \%$ lidocaine and infiltration of the periosteum of the target pedicle or pedicles with $0.25 \%$ bupivicaine. The control intervention comprised skin and subcutaneous tissue infiltration with $1 \%$ lidocaine and infiltration of the periosteum of the target pedicle or pedicles with $0.25 \%$ bupivicaine, similar to that administered during routine vertebroplasty. Pressure was applied to the back to simulate manipulation from vertebroplasty needles, and the methacrylate monomer was opened in the procedure room to simulate a vertebroplasty.

Follow-Up. Subjects and study coordinators performing follow-up interviews remained blinded to the procedure type. Subjects completed the study measures at predetermined time points up to 1 year. The primary outcomes were a numeric rating of pain on a scale from zero $=$ no pain to $10=$ pain as bad as could be and the modified Roland-Morris Disability Questionnaire, a measure of back pain - related physical disability ${ }^{13,14}$ at 30 days. Subjects were allowed to cross over to the other procedure after 30 days but remained blinded to procedure type for 1 year.

Study Groups. In the current report, we describe baseline characteristics of INVEST participants enrolled to date at the lead site. To better understand how eligible patients who enrolled versus those who did not enroll might differ, we compared the subgroup of patients who enrolled at the lead site with patients at the lead site who were eligible but who did not enroll. Such data about eligible nonenrolled patients were not available at the other sites. Because we did not perform prospective data collection in nonenrolled patients in the exact same fashion as was done for INVEST, data were typically limited to pain severity and scores on the modified Roland-Morris Disability Scale (RMDS). ${ }^{13,14}$

At each study site, enrolled patients were interviewed with study case report forms by a research coordinator. At the lead site, nonenrolled patients were interviewed as part of clinical practice by a nurse dedicated to the vertebroplasty practice. In clinical practice at the lead INVEST site, pain questions included queries about "pain at rest" and "pain with activity," whereas in the INVEST trial, patients were asked to verbally rate their average pain intensity during the preceding 24 hours on a scale of zero $=$ no pain to $10=$ pain as bad as could be.
Differences in pain questionnaires between the INVEST trial and the routine clinical practice arose because the study was designed before the principal investigator relocated to the current lead site. We, therefore, summarized the pain ratings made by study participants and those made by patients who did not enroll in the study, but we did not make any formal statistical comparison. Other outcomes measures, such as the SF-36, ${ }^{15}$ were not in routine clinical use at the lead site during the time of the INVEST study, so they were not available in the chart review.

\section{Statistical Analyses}

We calculated means and SDs for age, RMDS, and pain scores and compared study participants with eligible nonenrolled patients on each measure (except for pain scores) by using 2-sample $t$ tests, assuming unequal variances. We presented and compared the proportion of women for both groups by using $\chi^{2}$ tests. All statistical analyses were conducted by using STATA/IC 10.0 (StataCorp, College Station, Tex).

\section{Results}

Of the 972 patients screened at the lead site, 12\% $(n=119)$ were eligible for enrollment, with the most common reasons for exclusion being presence of tumor, infection, coagulopathy, and the absence of documented osteoporosis. Of the 119 meeting eligibility criteria, 27 (23\%) patients approached for study participation enrolled in the INVEST study. Of the 92 eligible patients who declined enrollment at the lead site, we had demographic, RMDS scores, and pain information on 70 patients. To date, we have enrolled 125 patients in INVEST at 10 sites in 4 countries.

Mean ages were similar across both comparison groups, averaging 73.7 years among enrolled participants and 76.6 years among nonenrolled patients $(P=.17$, Table). Approximately $75 \%$ of patients were women in both groups, with no significant difference between groups $(P=.64)$. Enrolled patients at the lead site did not differ from eligible nonenrolled patients at the lead site in RMDS scores (mean $=18.0 \pm 4.2$ versus $18.6 \pm 3.7, P=.51$ ). Among the enrolled patients, the mean "average pain intensity during the prior 24 hours" was $7.6 \pm 2.1$ among patients enrolled at the lead site. Among eligible nonenrolled patients at the lead site, the mean pain at rest was $3.4 \pm 3.3$ and the mean pain with activity was $8.5 \pm$ 2.0 . 


\section{Discussion}

Our data indicate that age, distribution of men versus women, and back pain - related disability, as measured by the RMDS, were similar between enrolled and nonenrolled patients at the lead site in INVEST. Mean RMDS scores did not differ between groups, as judged by either a conventional test of statistical significance or by currently accepted definitions of clinically meaningful differences. ${ }^{16}$ These data suggest that outcomes from INVEST, yet to be reported, likely will be generalizable to typical vertebroplasty patients who have baseline characteristics that would have allowed enrollment in INVEST.

The modified RMDS was initially developed for the study of patients with low-back pain and sciatica. The original report of the RMDS compared pain severity descriptors with scores on this scale. ${ }^{13}$ Pain that was "almost unbearable" or "very bad" was associated with RMDS mean scores of 14-15, with $95 \%$ confidence intervals ranging from 14 to 19 . The mean score for enrolled patients in the INVEST trial currently is approximately $17-18$, indicating both severe functional disability and pain levels.

Unfortunately, we are unable to compare directly baseline pain scores across groups. Patients enrolled in the INVEST trial responded to an ordinal $0-10$ pain question describing "average pain over the past 24 hours," whereas nonenrolled patients responded to a question posed as "pain at rest" and "pain with activity." This disparity concerning how pain questions are posed to vertebroplasty patients has received little, if any, attention previously. Indeed, apart from studies published by the lead INVEST site in which specific pain questions about rest and activity are reported, ${ }^{14,17}$ we were unable to find any previous vertebroplasty publication that describes exactly how pain-related questions are asked. Previous studies have shown that simply asking for pain at rest versus with activity will change the mean baseline pain by $4-5$ points on a 10 point scale. ${ }^{14,17}$ Further modifiers such as "average pain over the past 24 hours" as in the INVEST trial likely will modulate the reported pain level, compared with series that ask for worst pain. Unfortunately, given the dearth of detailed information concerning the pain measures used in previous publications, we cannot directly compare the INVEST baseline pain data with pain data in other studies. We surmise, however, that an "average" pain of approximately 7 of 10 indicates pain severity comparable with the level of 7-9 of 10 in prior studies. ${ }^{1-9}$

We have previously reported baseline RMDS data from a consecutive group of vertebroplasty patients treated at the lead site for INVEST but entirely separate from the INVEST trial. ${ }^{14}$ The mean RMDS score for this previously reported cohort was approximately 18 , similar to that of the overall enrolled cohort and nearly exactly the same as that of enrolled patients at the lead site. This finding lends further credence to the idea that patients enrolled in INVEST are at least as disabled by their back pain as most typical vertebroplasty patients.
This study has several limitations. Although the comparison group of nonenrolled patients was treated at the lead INVEST site concurrent with the enrolled cohort, data collection schemes for the enrolled-versus-nonenrolled patients differed. Enrolled patients were interviewed with dedicated case report forms by a research coordinator. Nonenrolled patients were interviewed as part of clinical practice by a nurse dedicated to the vertebroplasty practice, with different questions used to assess pain. Another limitation is that RMDS scores were available for eligible nonenrolled patients only at the lead site. Despite these limitations, the data presented here indicate that the pain and disability experienced by enrolled INVEST patients mimicked that of nonenrolled eligible patients.

\section{References}

1. Barbero S, Casorzo I, Durando M, et al. Percutaneous vertebroplasty: the follow-up. Radiol Med 2008;113:101-13

2. Do HM, Kim BS, Marcellus ML, et al. Prospective analysis of clinical outcomes after percutaneous vertebroplasty for painful osteoporotic vertebral body fractures. AJNR Am J Neuroradiol 2005;26:1623-28

3. Kobayashi K, Shimoyama K, Nakamura K, et al. Percutaneous vertebroplasty immediately relieves pain of osteoporotic vertebral compression fractures and prevents prolonged immobilization of patients. Eur Radiol 2005; 15:360-67

4. Krauss M, Hirschfelder $\mathrm{H}$, Tomandl B, et al. Kyphosis reduction and the rate of cement leaks after vertebroplasty of intravertebral clefts. Eur Radiol 2006;16:1015-21

5. McGraw JK, Lippert JA, Minkus KD, et al. Prospective evaluation of pain relief in 100 patients undergoing percutaneous vertebroplasty: results and followup. J Vasc Interv Radiol 2002;13:883-86

6. Peh WC, Gilula LA, Peck DD. Percutaneous vertebroplasty for severe osteoporotic vertebral body compression fractures. Radiology 2002;223:121-26

7. Singh AK, Pilgram TK, Gilula LA. Osteoporotic compression fractures: outcomes after single- versus multiple-level percutaneous vertebroplasty. Radiology 2006;238:211-20

8. Zoarski GH, Snow P, Olan WJ, et al. Percutaneous vertebroplasty for osteoporotic compression fractures: quantitative prospective evaluation of long-term outcomes. J Vasc Interv Radiol 2002;13:139-48

9. Alvarez L, Alcaraz M, Perez-Higueras A, et al. Percutaneous vertebroplasty: functional improvement in patients with osteoporotic compression fractures. Spine 2006;31:1113-18

10. Diamond TH, Champion B, Clark WA. Management of acute osteoporotic vertebral fractures: a nonrandomized trial comparing percutaneous vertebroplasty with conservative therapy. Am J Med 2003;114:257-65

11. Voormolen MH, Mali WP, Lohle PN, et al. Percutaneous vertebroplasty compared with optimal pain medication treatment: short-term clinical outcome of patients with subacute or chronic painful osteoporotic vertebral compression fractures-The VERTOS Study. AJNR Am J Neuroradiol. 2007;28:555-60

12. Gray LA, Jarvik JG, Heagerty PJ, et al. Investigational vertebroplasty efficacy and safety trial (INVEST): a randomized controlled trial of percutaneous vertebroplasty. BMC Musculoskelet Disord 2007;8:126

13. Roland M, Morris R. A study of the natural history of back pain. Part I. Development of a reliable and sensitive measure of disability in low-back pain. Spine 1983;8:141-44

14. Trout AT, Kallmes DF, Gray LA, et al. Evaluation of vertebroplasty with a validated outcome measure: the Roland-Morris Disability Questionnaire. AJNR Am J Neuroradiol 2005;26:2652-57

15. Ware JE, Kosinski M, Dewery JE. How to Score Version 2 of the SF-36 Health Survey. Lincoln, RI: QualityMetric; 2000

16. Lauridsen $\mathrm{HH}$, Hartvigsen J, Manniche $\mathrm{C}$, et al. Responsiveness and minimal clinically important difference for pain and disability instruments in low back pain patients. BMC Musculoskelet Disord 2006;7:82

17. Layton KF, Thielen KR, Koch CA, et al. Vertebroplasty, first 1000 levels of a single center: evaluation of the outcomes and complications. AJNR Am J Neuroradiol 2007;28:683-89 